\title{
O papel do profissional da Atenção Primária à Saúde em cuidados paliativos
}

\author{
The role of Primary Health Care professionals in palliative care \\ El rol del profesional de la Atención Primaria de Salud en cuidados paliativos
}

Mariana Lobato dos Santos Ribeiro Silva. Fundação Faculdade de Medicina (FFM). São Paulo, SP, Brasil. marianalobatorb@gmail.com (Autora correspondente)

\section{Resumo}

Introdução: 0 cuidado a pacientes em fase final da vida é cada vez mais frequente nos serviços de saúde devido ao progressivo envelhecimento da população e o consequente aumento de pacientes com doenças graves. No Brasil, os poucos serviços de cuidados paliativos geralmente estão ligados a hospitais especializados, mas, em vários países, a Atenção Primária à Saúde (APS) é considerada o melhor nível de assistência para a prestação e coordenação dos cuidados paliativos de seus usuários. Métodos: Neste estudo realizou-se uma revisão integrativa de literatura, objetivando compreender quais os papéis dos profissionais da APS em cuidados paliativos. Resultados: Os textos selecionados foram submetidos à análise temática, emergindo oito categorias que descrevem o papel dos profissionais de APS em cuidados paliativos e evidenciam que sua participação é essencial: 1) Possibilitar o cuidado domiciliar; 2) Garantir assistência com integralidade; 3) Utilizar a longitudinalidade como ferramenta de cuidado; 4) Responsabilizar-se pelo cuidado paliativo com a família; 5) Buscar aprimoramento profissional em cuidados paliativos; 6) Viabilizar a coordenação do cuidado; 7) Facilitar 0 acesso do paciente a cuidados paliativos e sua família; 8) Trabalhar em parcerias dentro e fora da APS. Conclusão: 0 acesso facilitado a cuidados paliativos, próximo ao lar, associado ao manejo constante dos sintomas e à sensibilidade para com a realidade das famílias, faz toda a diferença para os pacientes em fase final da vida.

\section{Abstract}

Background: Patients in end of life care are becoming increasingly common in health services owing to the population's progressive aging and the consequent increase in the number of patients with serious illnesses. In Brazil, the few existing palliative care services are usually linked to specialized hospitals; however, in many countries, Primary Health Care (PHC) is considered the best healthcare level for providing and coordinating palliative care to patients. Methods: An integrative literature review was carried out with the objective of understanding PHC practitioners' roles in palliative care. Results: The articles selected underwent thematic analysis resulting in eight categories describing the role of PHC professionals and their essential participation in palliative care: 1) Facilitate home care; 2) Ensure comprehensive assistance; 3) Use longitudinality as a tool of care; 4) Take responsibility for palliative care with the family; 5) Seek further professional development in palliative care; 6) Enable the coordination of palliative care; 7) Facilitate the access to palliative care to the patients and their families; 8) Work collaboratively inside and outside PHC. Conclusion: Easy near-home access to palliative care associated with constant management of symptoms and with sensibility to the reality of families is extremely important and helpful to patients in end of life stage.

\section{Resumen}

Introducción: La atención a los pacientes en la etapa final de la vida es cada vez más común en los servicios de salud debido al progresivo envejecimiento de la población y al consiguiente aumento de pacientes con enfermedades graves. En Brasil, los pocos servicios de cuidados paliativos suelen estar vinculados a hospitales especializados, pero en muchos países la Atención Primaria de Salud (APS) es considerada el mejor nivel de atención para la prestación y coordinación de los cuidados paliativos de sus usuarios. Métodos: Se realizó una revisión integradora de la literatura con el objetivo de comprender las funciones de los profesionales de la APS en cuidados paliativos. Resultados: Los artículos seleccionados se sometieron a análisis temático, surgiendo ocho categorías que describen el papel de los profesionales de la APS en cuidados paliativos y que evidencian su esencial participación: 1) Permitir el cuidado en el hogar; 2) Garantizar la asistencia integral; 3) Utilizar la longitudinalidad como herramienta del cuidado; 4) Asumir la responsabilidad de los cuidados paliativos con la familia; 5) Buscar el desarrollo profesional en los cuidados paliativos; 6) Facilitar la coordinación del cuidado; 7) Facilitar el acceso del paciente y su familia a los cuidados paliativos; 8) Trabajar en colaboración dentro y fuera de la APS. Conclusión: El fácil acceso a los cuidados paliativos, próximo al hogar, asociado al manejo constante de los síntomas y la sensibilidad con la realidad de las familias es de gran importancia para los pacientes en la etapa final de la vida.

Como citar: Silva MLSR. O papel do profissional da Atenção Primária à Saúde em cuidados

paliativos. Rev Bras Med Fam Comunidade. 2014;9(30):45-53.

Disponível em: http://dx.doi.org/10.5712/rbmfc9(30)718
Palavras-chave:

Atenção Primária à Saúde

Cuidados Paliativos

Saúde da Família

\section{Keywords:}

Primary Health Care

Palliative Care

Family Health

Palabras clave: Atención Primaria de Salud Cuidados Paliativos Salud de la Familia

Fonte de financiamento:
declara não haver.
Parecer CEP:
dada a natureza deste estudo
não foi necessária a aprovação
por Comitê de Ética em
Pesquisa (CEP).
Conflito de interesses:
declara não haver.
Recebido em: 13/03/2013
Aprovado em: 16/09/2013




\section{Introdução}

O cuidado a pacientes em fase final das suas vidas é, cada vez mais, uma realidade no cotidiano dos profissionais de saúde nos vários níveis de assistência. Isso se deve ao progressivo envelhecimento da população e à maior sobrevida de portadores de doenças graves e fatais com evoluçáo progressiva. ${ }^{1,2}$ Sendo assim, torna-se cada vez mais urgente a priorizaçáo do provimento, organização e coordenação da prestação de cuidados adequados a essa população.

Cuidados Paliativos são uma abordagem de atenção à saúde que visa à melhoria da qualidade de vida para pacientes e famílias que enfrentam os problemas associados a doenças graves, progressivas e incuráveis. ${ }^{1}$ A Organização Mundial da Saúde (OMS), desde 2002, define Cuidado Paliativo como:

Uma abordagem que promove a qualidade de vida de pacientes e seus familiares que enfrentam doenças que ameacem a continuidade da vida, por meio da prevenção e do alívio do sofrimento. Requer identificação precoce, avaliação e tratamento da dor e outros problemas de natureza física, psicossocial e espiritual (p. 6). ${ }^{3}$

Desse modo, os conceitos de terminalidade e impossibilidade de cura perdem sua centralidade e fala-se em possibilidade ou não de tratamento modificador da doença, contrapondo a ideia de "não ter mais nada a se fazer". ${ }^{4}$

Não é possível relatar, mesmo que sucintamente, todas as necessidades de cuidados dessas pessoas e suas famílias, pois antes de tudo, é fundamental se ter em mente que cada um é produto de sua história de vida, valores, crenças, condição social e acesso à saúde. Desse modo, é fundamental que todos os envolvidos nessa tarefa enfrentem tal complexidade sem receios, para prestarem assistência integral a esses pacientes e seus familiares, nesse momento de maior fragilidade. ${ }^{5}$

Ainda assim, pacientes considerados fora de possibilidade de cura acumulam-se nos hospitais, recebendo muitas vezes assistência inadequada, ainda focada na tentativa de "salvar a vida", utilizando métodos invasivos e tecnologias duras. Essa abordagem ignora o sofrimento, não trata os sintomas mais prevalentes e frequentemente mantém o indivíduo isolado de sua família, de seu lar e de suas lembranças. A morte ocorre solitária. Náo se trata de incentivar uma atitude contrária à medicina tecnológica, mas questionar a centralidade do "curar" e o uso de tecnologias duras na prática e mesmo na formação dos profissionais de saúde. É, antes, um incentivo à reflexão sobre a conduta dos profissionais de saúde diante da finitude humana. ${ }^{4}$

No Brasil, ainda são poucos os serviços de cuidados paliativos, apesar de já estar promulgada legislação específica estabelecendo no âmbito do Sistema Único de Saúde (SUS) o cuidado paliativo e o atendimento/internação domiciliar. ${ }^{6}$ Observa-se o crescimento da oferta desses serviços ano a ano, ainda que a maioria desses esteja ligada a hospitais especializados e de ensino, por meio de internação, atendimento ambulatorial e domiciliar. ${ }^{5,7}$

Em vários países, entretanto, a Atenção Primária à Saúde (APS) é considerada o melhor nível de assistência à saúde para a prestação e coordenação dos cuidados paliativos de seus usuários. ${ }^{8-11}$ Isso porque se entende que a proximidade geográfica, cultural e emocional desses profissionais pode contribuir em muito para que o cuidado ocorra de forma humanizada, respeitando-se a autonomia de pacientes e famílias de forma coordenada, evitando-se a fragmentação do indivíduo a partir dos múltiplos especialistas que usualmente estão envolvidos nesses casos, devido à sua complexidade. ${ }^{12}$ Além disso, a APS pode estruturar-se de modo a privilegiar a permanência do paciente em cuidados paliativos no domicílio, evitando seu afastamento da família em seus momentos finais de vida. ${ }^{2}$

A APS é o locus de responsabilidade pela atenção à saúde de pacientes e suas famílias no decorrer do tempo. Além de oferecer o acesso ao sistema de saúde para todas as necessidades de seus usuários a APS acompanha suas histórias de vida, oferecendo atenção e cuidado integral, coordenando e integrando o atendimento prestado por outros serviços de saúde. Também compartilha algumas características com os outros níveis de assistência como atenção à prevenção, tratamento e reabilitação, assim como o trabalho em equipe. ${ }^{13}$

A Estratégia Saúde da Família (ESF) foi instituída no Brasil em 1994, objetivando a reorganização do modelo de assistência à saúde a partir da atenção primária, em conformidade com os princípios do SUS. Além disso, o Ministério da Saúde do Brasil afirma que a ESF deve: 
Prestar, na unidade de saúde e no domicílio, assistência integral, contínua, com resolubilidade e boa qualidade às necessidades de saúde da população adstrita; eleger a família e o seu espaço social como núcleo básico de abordagem no atendimento à saúde; humanizar as práticas de saúde através do estabelecimento de um vínculo entre os profissionais de saúde e a população; proporcionar o estabelecimento de parcerias através do desenvolvimento de açôes intersetoriais (p. 10). ${ }^{14}$

Sendo assim, fica claro que, no advento de ter sob sua responsabilidade uma família em que um de seus membros está (ou deveria estar) em cuidado paliativo, é de responsabilidade da equipe de ESF abordar esse cuidado. Ainda assim, com frequência pacientes no final da vida não são identificados como casos "da atenção primária" uma vez que se supóe que apenas casos clínicos "mais simples" possam ser acolhidos e conduzidos pelas equipes. Isso porque, inicialmente, esperava-se que as equipes de ESF focassem sua ação "sobre os fatores de risco aos quais a população está exposta" (p. 10), ${ }^{14}$ entendendo que doenças transmissíveis e doenças crônicas deveriam ser consideradas "prioridades”, assim como populaçóes específicas como crianças menores de dois anos e gestantes, por meio de açôes programáticas. Com o progressivo envelhecimento da população, entretanto, a realidade que se impóe para as equipes pode ser muito diferente.

Ainda que não existam no Brasil parâmetros oficiais para o cálculo das necessidades de Cuidados Paliativos, a OMS afirma que essa necessidade pode ser estimada a partir do perfil de mortalidade de uma população, pois, entre as mortes ocorridas por causa natural, $50 \%$ a $80 \%$ seriam passíveis de Cuidados Paliativos, considerando-se todos os diagnósticos. ${ }^{4}$ Fica evidente que existe uma enorme demanda a ser acolhida e cuidada. Apesar da crescente demanda e da complexidade dos casos (que muitas vezes requerem açôes, procedimentos e tecnologias que não são usuais nesse nível de assistência), ainda são raros os estudos brasileiros sobre quais açóes caberiam à APS em cuidados paliativos.

Com essa questão em mente realizou-se esta revisão de literatura objetivando compreender: qual o papel dos profissionais de atenção primária à saúde em cuidados paliativos?

\section{Métodos}

Os seguintes passos do método da revisão integrativa da literatura foram seguidos: a identificação do problema (foi definido claramente o propósito da revisão); a busca da literatura (com a delimitação de palavras-chave, bases de dados e aplicação dos critérios definidos para a seleção dos artigos); a avaliação e a análise dos dados obtidos. ${ }^{15-17}$

A busca dos estudos ocorreu no período de junho a julho de 2012. Os critérios de inclusão dos estudos foram: artigos em português, inglês e espanhol, publicados nos últimos cinco anos, que apresentassem em sua discussão consideraçôes sobre o papel dos profissionais de APS em cuidados paliativos, indexados nas bases de dados LILACS e PUBMED.

Para a realização da busca, foram utilizadas combinaçôes entre as seguintes palavras-chave, consideradas descritores no DeCS (Descritores em Ciências de Saúde) e MeSH (Medical Subject Headings): Enfermagem Familiar (Family Nursing); Programa Saúde da Família (Family Health Program); Saúde da Família (Family Health); Medicina de Família e Comunidade (Family Practice); Atenção Primária à Saúde (Primary Care); Relaçôes Profissional-Família (Professional-Family Relations); Atitude diante da Morte (Attitude to Death); Cuidados paliativos (Palliative Care). Os termos foram cruzados como descritores e também como palavras do título e do resumo.

Nesta busca, foram inicialmente identificados 12 artigos científicos na base de dados LILACS e 213 artigos na base PUBMED para a leitura exploratória dos resumos e, entâo, selecionados 19 que foram lidos integralmente. Depois da leitura analítica destes artigos, 17 foram selecionados como objeto de estudo, ${ }^{1,2,-11,18-27}$ por apresentarem aspectos que respondiam à questão norteadora desta revisão. As etapas deste processo estão descritas no Quadro 1. O total de artigos selecionados na tabela é superior a 17, pois três artigos aparecem em cruzamentos de palavras-chave distintos. Os textos selecionados foram posteriormente submetidos à análise temática. 
Quadro 1. Distribuição das referências bibliográficas obtidas nas bases de dados Pubmed e Lilacs, segundo as palavras-chave selecionadas, Brasil, 2012.

\begin{tabular}{|c|c|c|c|c|c|}
\hline Base de Dados & $\begin{array}{l}\text { Palavras-chave cruzadas concomitantemente } \\
\text { (como palavras do resumo e como descritores) }\end{array}$ & $\begin{array}{c}\text { Número de } \\
\text { Referências } \\
\text { obtidas }\end{array}$ & $\begin{array}{c}\text { Resumos } \\
\text { Analisados }\end{array}$ & $\begin{array}{c}\text { Referências } \\
\text { selecionadas } \\
\text { para Análise }\end{array}$ & $\begin{array}{l}\text { Selecionados } \\
\text { para Revisão }\end{array}$ \\
\hline \multirow[t]{10}{*}{ Lilacs } & Enfermagem familiar/Atenção Primária à Saúde/Cuidados paliativos & 0 & 0 & 0 & 0 \\
\hline & Enfermagem familiar/Atenção Primária à Saúde/Atitudes diante da Morte & 0 & 0 & 0 & 0 \\
\hline & PSF/Cuidados paliativos & 0 & 0 & 0 & 0 \\
\hline & PSF/Atitudes diante da Morte & 0 & 0 & 0 & 0 \\
\hline & Medicina de Família e Comunidade/Atitudes diante da Morte & 0 & 0 & 0 & 0 \\
\hline & Medicina de Família e Comunidade/Cuidados paliativos & 4 & 4 & 2 & 2 \\
\hline & Saúde da Família/Atenção Primária à Saúde/Cuidados paliativos & 1 & 1 & 1 & 1 \\
\hline & Saúde da Família/Atenção Primária à Saúde/Atitudes diante da Morte & 0 & 0 & 0 & 0 \\
\hline & Relações profissional-família/Atenção Primária à Saúde/Cuidados paliativos & 13 & 7 & 4 & 2 \\
\hline & Relações profissional-família/Atenção Primária à Saúde/Atitudes diante da Morte & 0 & 0 & 0 & 0 \\
\hline \multirow[t]{8}{*}{ Pubmed } & Family Nursing/Primary Care/Palliative Care & 0 & 0 & 0 & 0 \\
\hline & Family Nursing/Primary Care/Attitude to Death & 0 & 0 & 0 & 0 \\
\hline & Family Health/Primary Care/Palliative Care & 0 & 0 & 0 & 0 \\
\hline & Family Health/Primary Care/Attitude to Death & 0 & 0 & 0 & 0 \\
\hline & Family Practice/ Palliative Care & 0 & 0 & 0 & 0 \\
\hline & Family Practice/ Attitude to Death & 72 & 72 & 3 & 3 \\
\hline & Primary Care/ Professional-Family Relations/ Palliative Care & 126 & 93 & 7 & 9 \\
\hline & Primary Care/ Professional-Family Relations/ Attitude to Death & 24 & 24 & 3 & 3 \\
\hline
\end{tabular}

A análise temática consiste em um método para a identificaçáo, análise e registro de padróes (temas) a partir dos dados, organizando-os e descrevendo-os em detalhes. ${ }^{16} \mathrm{Um}$ tema deve capturar algo importante nos dados, que se relacione à pergunta da pesquisa e que represente um nível de resposta ou significado que se apresente como padrão nos dados. Entretanto, não se deve considerar um tema apenas por este estar presente com determinada frequência no material obtido, e sim, por meio da interpretação desse material pelo pesquisador. ${ }^{16,17}$

Os temas identificados pelo pesquisador podem ser formulados de modo dedutivo ou indutivo. Em uma abordagem indutiva os temas identificados estão fortemente ligados aos dados, enquanto em uma abordagem dedutiva o referencial teórico e os interesses analíticos do pesquisador determinam os temas a serem identificados. ${ }^{16}$ Fereday e Muir-Cochrane ${ }^{17}$ afirmam que é possível uma abordagem analítica que utilize um processo híbrido, utilizando temas desenvolvidos de forma indutiva e dedutiva, a partir do referencial teórico utilizado pelo autor.

Neste estudo, adotou-se o processo híbrido de formulação dos temas, tendo como referencial teórico o livro de Barbara Starfield: Atenção Primária: equilíbrio entre necessidades de saúde, serviços e tecnologia, de $2002 .{ }^{13}$

\section{Resultados}

No Quadro 2 são apresentados os artigos selecionados para essa revisão, com autores, data e país de publicação, os sujeitos de pesquisa e as principais conclusão do estudo.

$\mathrm{Da}$ análise do conteúdo das publicaçóes, emergiram oito categorias temáticas: 1) Possibilitar o cuidado domiciliar; 2) Garantir assistência com integralidade; 3) Utilizar a longitudinalidade como ferramenta de cuidado; 4) Responsabilizar-se pelo cuidado paliativo com a família; 5) Buscar aprimoramento profissional em cuidados paliativos; 6) Viabilizar a coordenação do cuidado; 7) Facilitar o acesso do paciente em cuidados paliativos e sua familia; 8) Trabalhar em parcerias dentro e fora da APS. 
Quadro 2. Distribuição das referências incluídas na revisão integrativa, de acordo com ano de publicação, país, autores e tipo de estudo, Brasil, 2012.

\begin{tabular}{|c|c|c|c|c|c|}
\hline $\mathrm{N}^{0 *}$ & Ano e País & Autor & Sujeitos de Pesquisa & Tipo de estudo & Principais Conclusões \\
\hline 1 & 2010, Alemanha & $\begin{array}{l}\text { Schneider N, } \\
\text { Lueckmann SL, } \\
\text { Kuehne SLF, et al. }\end{array}$ & $\begin{array}{l}\text { Especialistas em } \\
\text { Saúde Pública e } \\
\text { Cuidados Paliativos }\end{array}$ & $\begin{array}{l}\text { Qualitativo, descritivo- } \\
\text { exploratório }\end{array}$ & $\begin{array}{l}\text { Identificados seis aspectos mais relevantes para iniciativas de } \\
\text { saúde pública em cuidados paliativos: abordagem de cuidados } \\
\text { paliativos, o paciente e a família, os serviços de saúde, } \\
\text { informação e capacitação, pesquisa e financiamento. }\end{array}$ \\
\hline 2 & 2007, Brasil & $\begin{array}{l}\text { Florianí CA, } \\
\text { Schramm SR. }\end{array}$ & & Reflexão teórica & $\begin{array}{l}\text { A reflexão sobre o papel da equipe da atenção primária com } \\
\text { respeito aos cuidados paliativos poderá contribuir para o } \\
\text { aprimoramento e para a difusão desta disciplina de cuidados no } \\
\text { fim da vida. }\end{array}$ \\
\hline 7 & 2010, Brasil & $\begin{array}{l}\text { Rabelo CAFG, } \\
\text { Rodrigues PHA }\end{array}$ & $\begin{array}{l}\text { Famílias relacionadas } \\
\text { ao Programa } \\
\text { Assistência Domiciliar } \\
\text { Integrada, Rio de } \\
\text { Janeiro, RJ. }\end{array}$ & $\begin{array}{l}\text { Qualitativo, descritivo- } \\
\text { exploratório }\end{array}$ & $\begin{array}{l}\text { A organização dos cuidados domiciliares a partir da atenção } \\
\text { primária, articulada aos outros níveis de complexidade exigiria } \\
\text { integrar tecnologia e humanização, a partir do encontro da } \\
\text { tecnologia "dura" com a tecnologia "leve". }\end{array}$ \\
\hline 8 & 2007, Holanda & $\begin{array}{l}\text { Borgsteede SD, } \\
\text { Deliens L, } \\
\text { Wal GVD, et al. }\end{array}$ & Médicos de família & $\begin{array}{l}\text { Quantitativo, descritivo- } \\
\text { exploratório }\end{array}$ & $\begin{array}{l}\text { Em pacientes de cuidados paliativos, a cooperação interdisciplinar } \\
\text { dos médicos de família com outros profissionais é altamente } \\
\text { prevalente, especialmente com cuidadores informais. A } \\
\text { cooperação é mais prevalente em pacientes mais jovens, nos } \\
\text { pacientes com câncer e se o atendimento psicossocial for } \\
\text { importante para o cuidado. }\end{array}$ \\
\hline 9 & 2007, Canadá & $\begin{array}{l}\text { Barnes EA, Fan G, } \\
\text { Harris } K \text {, et al. }\end{array}$ & $\begin{array}{l}\text { Pacientes de um } \\
\text { serviço de radioterapia }\end{array}$ & $\begin{array}{l}\text { Quantitativo, descritivo- } \\
\text { exploratório }\end{array}$ & $\begin{array}{l}\text { Menos da metade dos pacientes pesquisados percebem seu } \\
\text { médico de família envolvido em seu tratamento de câncer. }\end{array}$ \\
\hline 10 & 2010, Holanda & $\begin{array}{l}\text { Verhoeven AAH, } \\
\text { Schuling J, } \\
\text { Maeckelber ELM. }\end{array}$ & $\begin{array}{c}\text { Residentes de } \\
\text { Medicina de Família }\end{array}$ & $\begin{array}{l}\text { Qualitativo, descritivo- } \\
\text { exploratório }\end{array}$ & $\begin{array}{l}\text { Ao lidarem com a proximidade da morte de seus pacientes, a } \\
\text { relação única entre médico e paciente se transforma, assim como } \\
\text { os valores desses médicos. }\end{array}$ \\
\hline 11 & 2007, Inglaterra & $\begin{array}{l}\text { Walshe C, Todd C, } \\
\text { Caress AL, et al. }\end{array}$ & Clínicas de APS & $\begin{array}{l}\text { Qualitativo, descritivo- } \\
\text { exploratório }\end{array}$ & $\begin{array}{l}\text { Futuras políticas e protocolos de cuidados paliativos devem } \\
\text { ter em conta o trabalho colaborativo entre diferentes níveis de } \\
\text { assistência. }\end{array}$ \\
\hline 18 & $\begin{array}{c}\text { 2010, } \\
\text { Dinamarca }\end{array}$ & $\begin{array}{l}\text { Neergaard MA, } \\
\text { Vedstesd } \mathrm{P} \text {, } \\
\text { Olesen } \mathrm{F} \text {, et al. }\end{array}$ & $\begin{array}{l}\text { Médicos de família } \\
\text { que atenderam } \\
\text { pacientes em cuidado } \\
\text { paliativo domiciliar }\end{array}$ & $\begin{array}{l}\text { Quantitativo, descritivo- } \\
\text { exploratório }\end{array}$ & $\begin{array}{l}\text { A morte domiciliar aumenta a percepção das famílias enlutadas } \\
\text { da trajetória de cuidado paliativo como bem-sucedida. } 0 \text { impacto } \\
\text { do contato com médico de família pareceu ser importante, mas } \\
\text { ainda necessita mais investigação. }\end{array}$ \\
\hline 19 & 2008, Canadá & $\begin{array}{l}\text { Marshall D, Howell D, } \\
\text { Brazil K, et al. }\end{array}$ & Médicos de família & $\begin{array}{l}\text { Qualitativo, Estudo de } \\
\text { Caso }\end{array}$ & $\begin{array}{l}\text { Os médicos participantes relataram melhora da comunicação, } \\
\text { colaboração interprofissional eficaz, e a capacidade de prestar } \\
\text { cuidados paliativos em casa, para seus pacientes no final de vida } \\
\text { na comunidade. }\end{array}$ \\
\hline 20 & 2010, EUA & Han PKJ, Rayson D. & Literatura científica & Revisão de & $\begin{array}{l}\text { É claramente necessário desenvolver e avaliar intervenções para } \\
\text { coordenar o tratamento do câncer no final da vida assim como } \\
\text { promover a integração da atenção primária e oncologia. }\end{array}$ \\
\hline 21 & 2009, Brasil & $\begin{array}{l}\text { Valente SH, } \\
\text { Teixeira MB }\end{array}$ & Enfermeiros do PSF & $\begin{array}{l}\text { Pesquisa qualitativa, } \\
\text { descritiva-exploratória }\end{array}$ & $\begin{array}{l}\text { Apesar de ser uma experiência recompensadora, o cuidado } \\
\text { paliativo domiciliar no contexto do PSF é difícil e desgastante. }\end{array}$ \\
\hline 22 & 2007, Brasil & $\begin{array}{l}\text { Benedetto MAC, } \\
\text { Castro AG, } \\
\text { Carvalho } E \text {, et al. }\end{array}$ & $\begin{array}{l}\text { Residentes de } \\
\text { Medicina de Família e } \\
\text { Comunidade }\end{array}$ & Relato de Experiência & $\begin{array}{l}\text { A abordagem narrativa usada como estratégia didática em } \\
\text { cuidados paliativos enriquece a prática e é coerente com os } \\
\text { preceitos da medicina familiar. }\end{array}$ \\
\hline 23 & 2008, Inglaterra & $\begin{array}{c}\text { Shipman C, Gysels M, } \\
\text { White P, et al. }\end{array}$ & $\begin{array}{l}\text { Profissionais de } \\
\text { saúde, acadêmicos e } \\
\text { usuários da Atenção } \\
\text { Primária e Secundária, } \\
\text { especialistas em }\end{array}$ & $\begin{array}{c}\text { Estudo qualitativo, } \\
\text { descritivo-exploratório }\end{array}$ & $\begin{array}{l}\text { As definições de final da vida e cuidados paliativos necessitam de } \\
\text { esclarecimentos e padronização. Também é necessária a definição } \\
\text { de modelos de boas práticas e o compromisso de fornecer } \\
\text { recursos para prestação de serviços adequada e formação } \\
\text { profissional. }\end{array}$ \\
\hline
\end{tabular}


Quadro 2. Distribuição das referências incluídas na revisão integrativa, de acordo com ano de publicação, país, autores e tipo de estudo, Brasil, 2012.

Continuação...

\begin{tabular}{|c|c|c|c|c|c|}
\hline $\mathrm{N}^{0 *}$ & Ano e País & Autor & Sujeitos de Pesquisa & Tipo de estudo & Principais Conclusões \\
\hline 24 & Brasil, 2010 & $\begin{array}{l}\text { Pinheiro TRP, } \\
\text { Benedetto MAC, } \\
\text { Levites MR, et al. }\end{array}$ & $\begin{array}{l}\text { Residentes e } \\
\text { preceptores de } \\
\text { Medicina de Família e } \\
\text { Comunidade }\end{array}$ & $\begin{array}{l}\text { Estudo qualitativo, } \\
\text { descritivo-exploratório. }\end{array}$ & $\begin{array}{l}\text { As atividades relacionadas ao cuidado paliativo possibilitaram } \\
0 \text { aprendizado sobre a dor, o sofrimento e o enfrentamento da } \\
\text { morte, eventos que fazem parte da vida humana, tão naturalmente } \\
\text { quanto possível, mas sem perder uma atitude respeitosa. }\end{array}$ \\
\hline 25 & 2007, Austrália & McGrath P. & $\begin{array}{l}\text { Médicos de família e } \\
\text { comunidade }\end{array}$ & $\begin{array}{l}\text { Estudo qualitativo, } \\
\text { descritivo-exploratório. }\end{array}$ & $\begin{array}{l}0 \text { estudo indica que médicos de família querem estar envolvidos } \\
\text { ao longo do continuum de cuidados paliativos e, devido a } \\
\text { conhecimento holístico do paciente e sua família, estão bem } \\
\text { colocados para assegurar a liderança na coordenação dos } \\
\text { cuidados de base comunitária. }\end{array}$ \\
\hline 26 & 2007, Holanda & $\begin{array}{l}\text { Groot MM, Vernooij- } \\
\text { Dassen MLFL, } \\
\text { Verhagen SCA, et al. }\end{array}$ & $\begin{array}{l}\text { Médicos de família e } \\
\text { comunidade }\end{array}$ & $\begin{array}{c}\text { Quantitativo, descritivo- } \\
\text { exploratório }\end{array}$ & $\begin{array}{l}\text { Existe uma grande necessidade de, primeiramente, superar as } \\
\text { barreiras dentro da organização e coordenação de cuidados } \\
\text { paliativos na atenção primária. }\end{array}$ \\
\hline 27 & 2010, Noruega & $\begin{array}{l}\text { Johansen ML, } \\
\text { Holtedahl KA, } \\
\text { Rudebeck CE. }\end{array}$ & $\begin{array}{l}\text { Médicos de família e } \\
\text { comunidade }\end{array}$ & $\begin{array}{l}\text { Estudo qualitativo, } \\
\text { descritivo-exploratório }\end{array}$ & $\begin{array}{l}\text { Os entrevistados vêm seu lugar no tratamento do câncer como } 0 \\
\text { estar perto de seus pacientes. Entre as várias tarefas relatadas } \\
\text { encontramos três aspectos principais: a mediação, a prática do } \\
\text { cuidado e o cuidado pessoal. }\end{array}$ \\
\hline
\end{tabular}

${ }^{*}$ Conforme a numeração nas Referências Bibliográficas.

O primeiro tema identificado, Possibilitar o cuidado domiciliar, reflete a percepçáo de que a permanência do paciente em cuidado paliativo junto à sua família, no lar, é essencial e sinal de paliação com qualidade. Os autores dos textos analisados são enfáticos ao afirmar que o domicílio é o local preferencial para a realização dos cuidados paliativos e que a APS, devido à sua proximidade geográfica, devido ao seu conhecimento da realidade das famílias envolvidas e o vínculo já estabelecido, é o nível de assistência à saúde que pode possibilitar o cuidado nesse locus. ${ }^{918} \mathrm{O}$ paciente e sua família frequentemente manifestam interesse que a morte ocorra no domicílio. A ocorrência da morte no lar pode, inclusive, ser considerada um indicador de qualidade dos cuidados paliativos. Os autores afirmam que pacientes atendidos por equipes de APS têm maior probabilidade de falecerem em suas casas, pelo apoio clínico e emocional que recebem à sua decisão. ${ }^{119-21}$

Garantir a assistência com integralidade manifesta a preocupação dos autores com o risco de fragmentação da assistência de pacientes em cuidados paliativos, devido aos múltiplos especialistas e serviços de emergência que acompanham esses casos. $^{20-23} \mathrm{O}$ olhar ampliado característico da APS pode identificar demandas que ficaram despercebidas pelo olhar parcial, afastado do cotidiano das comunidades, dos serviços de atenção secundária e emergência, integrando a ação dos especialistas e adequando as orientaçóes à realidade sociocultural da família., ${ }^{9,21,24}$

Utilizar a longitudinalidade como ferramenta de cuidado apresenta mais uma potencialidade da APS ressaltada pelos autores dos textos analisados. O conhecimento das famílias, por vezes prévio ao diagnóstico de doença grave e fatal, leva os profissionais de APS a compreenderem suas dificuldades e as potencialidades em cada situação. ${ }^{1,19,25}$ A manutenção do acompanhamento por profissionais da APS aos pacientes em cuidado especializado facilita a transição para os cuidados paliativos e contribui nas tomadas de decisão posteriores. ${ }^{9,19,21,24}$

Os textos analisados trazem também que Responsabilizar-se pelo cuidado paliativo com a família leva os profissionais de APS a colocarem-se lado a lado com as famílias, agindo como tradutores do universo de exames, especialistas e laudos dos serviços especializados envolvidos nos tratamentos e paliaçáa..$^{20,27}$ Os profissionais de APS também atuam orientando as famílias nas questôes legais relacionadas ao adoecimento, como benefícios sociais e aposentadorias. ${ }^{22} \mathrm{~A}$ família é frequentemente identificada como cuidadora, mas a APS vê a própria família como objeto do cuidado, buscando apoiá-la no exercício do cuidado com seu ente em processo de morrer, buscando fortalecê-la e protegê-la da sobrecarga. ${ }^{1}$

Ao Buscar aprimoramento profissional em cuidados paliativos os profissionais de APS procuram suprir deficiências de sua formação hospitalocêntrica, focada em tecnologias duras e busca pela cura, não em qualidade de vida. ${ }^{11,25}$ Os autores declaram que desde a formação do profissional que atuará na APS é fundamental que se tenha experiências com paliação, garantindo o aprendizado desse novo paradigma de cuidado. ${ }^{24}$ 
Para que a resolubilidade da APS em cuidados paliativos seja satisfatória, os autores dos textos estudados declaram que é fundamental Trabalhar com parcerias dentro e fora da APS, com diferentes serviços e níveis de assistência à saúde. Os problemas de saúde desses pacientes e suas famílias são muito variados e, eventualmente, necessitam do suporte de tecnologias duras próprias de outros níveis de assistência. ${ }^{111}$ Além disso, devido à gravidade dos casos, esses pacientes e famílias demandam suporte 24 horas, tornando essencial o estabelecimento de parcerias com serviços de emergência e/ou a criaçáo de escalas entre outras equipes de APS, garantindo o atendimento fora do horário tradicional dos serviços de APS, mas ainda assim respeitando-se as decisôes e planos terapêuticos já estabelecidos entre a própria equipe de APS responsável e a família. 19,23,27

Viabilizar a coordenação do cuidado é, para os autores dos estudos analisados, uma das tarefas centrais da APS em cuidados paliativos, tendo em vista que, ao Trabalhar com parcerias, o papel de coordenaçáo do cuidado leva a Garantir a assistência com integralidade. Assim o profissional de APS deve manter-se atualizado sobre o tratamento especializado realizado por seus pacientes com doenças potencialmente fatais, buscando ativamente a contrarreferência, atuando como mediador entre

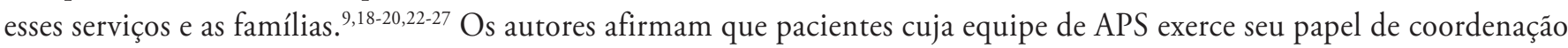
de cuidado eficazmente têm menos internaçôes, melhor manejo de sintomas e melhores escores de qualidade de vida..$^{20,26}$

Por fim, o tema Facilitar o acesso de pacientes em cuidados paliativos e suas famílias vem da percepção dos autores de que a APS pode atuar tanto como primeiro contato no manejo de sintomas físicos e emocionais como suporte para questôes relacionadas aos serviços especializados, laudos, encaminhamentos, etc., ${ }^{9,2,24}$ Para tanto, é fundamental que as agendas dos profissionais de APS estejam organizadas para suportar mais essa demanda de trabalho, com períodos de visita domiciliar e flexibilidade para receber familiares com dúvidas e angústias como demanda espontânea. ${ }^{9,25}$

\section{Discussão}

Dos oito temas identificados na análise temática da literatura selecionada, cinco relacionam-se diretamente com os atributos da APS descritos por Starfield: ${ }^{13}$ Acesso, Longitudinalidade, Integralidade, Coordenação e com o atributo derivado de Abordagem Familiar. Este fato reforça o argumento de que o cuidado paliativo e a atenção primária compartilham conceitos e princípios de trabalho que os tornam não só compatíveis, mas complementares.

$\mathrm{O}$ uso de instrumentos de abordagem familiar, frequentemente vistos em APS, como genogramas e ecomapas, facilita ao profissional conhecer as características da família atendida, identificar as características das relaçóes e antecipar possíveis dificuldades, além de contribuir com a comunicação e a mediação entre os membros da própria família em um momento de amplificação dos conflitos, para o planejamento de uma morte digna junto ao paciente em cuidados paliativos e sua família. ${ }^{12,28}$

A criação do Núcleo de Apoio à Saúde da Família (NASF) em $2008^{29}$ contribui para a manutençáo dos casos de cuidados paliativos na APS, tendo em vista que o suporte de psicólogos, nutricionistas, fonoaudiólogos, assistentes sociais, fisioterapeutas, terapeutas ocupacionais, geriatras entre outros profissionais aumenta a resolubilidade da equipe de APS e acrescenta em muito a gama de possibilidades de intervençôes e orientaçôes que podem ser oferecidas nesse nível de assistência.

Ainda assim, observa-se com frequência que os profissionais de APS/ESF enfrentam grandes dificuldades ao envolverem-se na paliação de seus pacientes e famílias: a falta de formação em cuidados paliativos, a falta de suporte especializado, especialmente onde o NASF ainda náo foi implementado, a desarticulação da rede de serviços de atenção à saúde no território, dificultando o estabelecimento de parcerias com serviços de emergência para o atendimento fora do horário de funcionamento das Unidades de Saúde da Família. Além disso, a sobrecarga de trabalho dificulta a necessária flexibilização das agendas para acolher as demandas nem sempre previsíveis desses pacientes e suas famílias. ${ }^{2}$

A Academia Nacional de Cuidados Paliativos (ANCP) define quatro níveis de atenção em cuidados paliativos:

- Ação paliativa - cuidado dispensado em nivel comunitário por equipe vinculada ao Programa Saúde da Família (PSF), treinada para tal finalidade;

- Cuidado Paliativo de grau I - cuidado dispensado por equipe especializada em Cuidados Paliativos em nivel hospitalar, ambulatorial ou domiciliar, porém sem leito próprio. Exemplo: equipe consultora em Cuidados Paliativos; 
- Cuidado Paliativo de grau II - cuidado dispensado por equipe especializada em Cuidados Paliativos em nivel hospitalar, ambulatorial ou domiciliar que atua com leitos próprios de internação. Equivalem tanto às enfermarias em hospitais gerais quanto às unidades hospitalares independentes;

- Cuidado Paliativo de grau III - mesma característica do cuidado de grau II acrescida de capacidade para a formação de profissionais em Cuidados Paliativos (p.83)4.

Nos dois primeiros níveis a APS tem um papel central, recebendo orientação dos serviços especializados quando necessário e coordenando a assistência. ${ }^{4}$ Nos demais níveis de cuidados paliativos, o cuidado é realizado predominantemente pelo serviço especializado mas a continuidade da assistência, como já apresentado nos resultados, pode garantir que o cuidado familiar e psicossocial seja mantido longitudinalmente e que as características socioculturais das famílias e pacientes sejam respeitadas.

Fica ainda a questão de se definirem quais os pacientes elegíveis para cuidados paliativos dentro da população adstrita atendida pelas equipes de APS. Alguns pesquisadores afirmam que devem ser considerados como candidatos para cuidados paliativos pacientes cujo profissional responsável responda "não" à pergunta "Você se surpreenderia se esse paciente morresse nos próximos 12 meses?". ${ }^{4}$ No Manual de Cuidados Paliativos, a ANCP afirma que: "O período de 12 meses é bastante restrito se pensarmos em tempo de vida, mas insuportavelmente longo se corresponder a tempo de sofrimento"(p. 26). ${ }^{4}$

\section{Considerações finais}

Os profissionais da APS têm o privilégio único de acompanharem seus usuários ao longo de toda sua vida, nas mais variadas situaçóes que levam a populaçáo a procurar os serviços de saúde. Da mesma forma que o nascimento é parte do cotidiano desses profissionais, sendo o ciclo gravídico-puerperal considerado prioridade para as ESF, o processo de morte também o é.

Os profissionais acompanham o envelhecimento e a fragilização de seus pacientes, os esforços das famílias para acomodarem as novas necessidades de cuidado de seus membros, os medos, a insegurança financeira e, por isso, não podem se esquivar nesse momento: é exatamente em situaçóes assim que toda a potencialidade das açóes da APS/ESF fica mais evidente. Nenhum outro serviço de saúde pode colocar-se lado a lado com essas famílias com tanta propriedade e enfrentar o caminho da paliaçáo com presença constante, orientação e acolhimento.

A partir dos temas que emergiram nesta revisão de literatura é possível declarar que, no trajeto percorrido por pacientes em final de vida e suas famílias, a atuação de qualidade da Atenção Primária à Saúde é mais importante do que nunca. O acesso facilitado, garantido, próximo ao lar, o manejo dos sintomas constantes e a sensibilidade e o respeito às realidades vivenciadas em nossas comunidades podem não mudar o desfecho clínico (a morte), mas certamente, o cuidado paliativo faz toda a diferença aos pacientes e seus familiares nessa etapa de finitude da vida.

\section{Referências}

1. Schneider N, Lueckmann SL, Kuehne SLF, Klindtworth K, Behmann M. Developing targets for public health initiatives to improve palliative care. BMC Public Health. 2010; 10:222. http://dx.doi.org/10.1186/1471-2458-10-222

2. Florianí CA, Schramm SR. Moral and operational challenges for the inclusion of palliative care in primary health care. Cad Saúde Pública. 2007; 23(9): 207280. http://dx.doi.org/10.1590/S0102-311X2007000900015

3. World Health Organization. Palliative care for older people: better practices. Denmark: WHO; 2011. Disponível em: http://www.euro.who.int/__data/ assets/pdf_file/0017/143153/e95052.pdf

4. Academia Nacional de Cuidados Paliativos (ANCP). Manual de cuidados paliativos. Rio de Janeiro: Diagraphic; 2009.

5. Conselho Regional de Medicina do Estado de São Paulo (CREMESP). Cuidado Paliativo. São Paulo: CREMESP; 2008.

6. Brasil. Lei n 10.424, de 15 de abril de 2002. Acrescenta capítulo e artigo à Lei n 8.080, de 19 de setembro de 1990, que dispõe sobre as condições para a promoção, proteção e recuperação da saúde, a organização e o funcionamento de serviços correspondentes e dá outras providências, regulamentando a assistência domiciliar no Sistema Único de Saúde. Diário Oficial da Repúblia Federativa do Brasil; Abr 2002. [acessado 2012 Jun 6]. Disponível em: http://www.planalto.gov.br/ccivil_03/leis/2002/110424.htm 
7. Rabelo CAFG, Rodrigues PHA. Saúde da Família e cuidados paliativos infantis: ouvindo os familiares de crianças dependentes de tecnologia. Ciênc Saúde Coletiva. 2010; 15(Supl. 2): 3157-66. http://dx.doi.org/10.1590/S1413-1232010000200013

8. Borgsteede SD, Deliens L, Wal GVD, Francke AL, Stalman WAB, Eijk JTME. Interdisciplinary cooperation of GPs in palliative care at home: A nationwide survey in the Netherlands. Scand J Prim Health Care, 2007. 25: 226-31. http://dx.doi.org/10.1080/02813430701706501

9. Barnes EA, Fan G, Harris K, Barbera L, Tsao M, Doyle M et al. Involvement of Family Physicians in the Care of Cancer Patients Seen in the Palliative Rapid Response Radiotherapy Program. J Clin Oncol. 2007; 25(36): 5758-62. http://dx.doi.org/10.1200/JCO.2007.13.3082

10. Verhoeven AAH, Schuling J, Maeckelber ELM. The death of a patient: a model for reflection in GP training. BMC Fam Pract. 2011; 12(2): 8-15. http:// dx.doi.org/10.1186/1471-2296-12-8

11. Walshe C, Todd C, Caress AL, Graham CC. Judgements about fellow professionals and the management of patients. Br J Gen Pract. 2008; $58: 264-72$. http://dx.doi.org/10.3399/bjgp08X279652

12. McDaniel SH, Campbell ThL, Hepworth J, Lorenz A. Family-Oriented Primary Care. New York: Springer; 2005.

13. Starlfield B. Atenção primária: equilíbrio entre necessidades de saúde, serviços e tecnologia. Brasília: UNESCO, Ministério da Saúde; 2002. Disponível em: http://bvsms.saude.gov.br/bvs/publicacoes/atencao_primaria_p1.pdf

14. Brasil. Ministério da Saúde. Secretaria de Assistência à Saúde. Coordenação de Saúde da Comunidade. Saúde da Família: uma estratégia para a reorientação do modelo assistencial. Brasília; 1997. Disponível em: http://bvsms.saude.gov.br/bvs/publicacoes/cd09_16.pdf

15. Whittemore R, Knafl K. The integrative review: updated methodology. J Adv Nurs. 2005; 52(5): 546-553. http://dx.doi.org/10.1111/j.13652648.2005.03621.x

16. Braun V, Clarke V. Using thematic analysis in psychology. Qual Res Psychol. 2006;(3): 77-101. http://dx.doi.org/10.1191/1478088706qp063oa

17. Fereday J, Muir-Cochrane E. Demonstrating Rigor using thematic analysis: a hybrid approach of inductie and deductive coding and theme development. Int J Qual Methods. 2006; 5(1): 8-15. http://dx.doi.org/10.1063/1.2011295

18. Neergaard MA, Vedstesd P, Olesen F, Solkolowski I, Jensen AB, Sondergaad J. Associations between successful palliative trajectories, place of death and GP involvement. Scand J of Prim Health Care. 2010; 28: 138-145. http://dx.doi.org/10.3109/02813432.2010.505316

19. Marshall D, Howell D, Brazil K, Howard M, Taniguchi A. Enhancing family physician capacity to deliver quality palliative home care: An end-of-life, shared-care model. Can Fam Physician. 2008; 54: 1703.e1-7 Disponível em: http://www.ncbi.nlm.nih.gov/pmc/articles/PMC2602612/?tool=pubmed

20. Han PKJ, Rayson D. The Coordination of Primary and Oncology Specialty Care at the End of Life. J Natl Cancer Inst Monogr. 2010; 40: 31-7. http:// dx.doi.org/10.1093/jncimonographs/lgq003

21. Valente SH, Teixeira MB. Estudo fenomenológico sobre a visita domiciliária do enfermeiro à família no processo de terminalidade. Rev Esc Enferm USP. 2009; 43(3): 665-61. http://dx.doi.org/10.1590/S0080-62342009000300022

22. Benedetto MAC, Castro AG, Carvalho E, Sanogo R, Blasco PG. From suffering to transcendence: Narratives in palliative care. Can Fam Physician. 2007; 53(8): 1277-79. Disponível em: http://www.cfp.ca/content/54/12/1703.full

23. Shipman C, Gysels M, White P, Worth A, Murray SA, Barclay S et al. Improving generalist end of life care: national consultation with practitioners, commissioners, academics, and service user groups. BMJ. 2008; 11: 337(7674). http://dx.doi.org/10.1136/bmj.a1720

24. Pinheiro TRP, Benedetto MAC, Levites MR, Giglio A, Blasco PG. Teaching Palliative Care to Residents and Medical Students. Fam Med. 2010; 42(8): 580-2. Disponível em: http://www.stfm.org/fmhub/fm2010/September/Thais\%20Raquel580.pdf

25. McGrath P. Care of the hematology patient and their family The GP viewpoint. Aust Fam Physician. 2007; 36(9): 779-81.

26. Groot MM, Vernooij-Dassen MLFL, Verhagen SCA, Crul BJP, Grol RPTM. Obstacles to the delivery of primary palliative care as perceived by GPs. Palliat Med. 2007; 21(8): 697-703. http://dx.doi.org/10.1177/0269216307083384

27. Johansen ML, Holtedahl KA, Rudebeck CE. A doctor close at hand: How GPs view their role in cancer care. Scand J Prim Health Care. 2010; $28: 249-55$. http://dx.doi.org/10.3109/02813432.2010.526792

28. Silva MCLSR, Silva L, Bousso RZ. A abordagem à família na Estratégia Saúde da Família: uma revisão integrativa da literatura. Rev Esc Enferm USP. 2011; 45(5): 1250-1255. http://dx.doi.org/10.1590/S0080-62342011000500031

29. Ministério da Saúde (BR). Portaria GM n 154, de 24 de janeiro de 2008. Cria os núcleos de Apoio à Saúde da Família - NASF. Diário Oficial da Repúblia Federativa do Brasil; 25 Jan 2008. [n. 18]. Disponível em: http://bvsms.saude.gov.br/bvs/saudelegis../gm/2008/prt0154_24_01_2008.html 